\title{
Optimal rule curves for interconnected reservoirs
}

\author{
A. Turgeon \\ Département de mathématiques et de génie industriel, \\ École Polytechnique de Montréal, Canada
}

\begin{abstract}
This paper shows that the optimal (or near-optimal) operating policy of a reservoir feeding a hydroelectric powerplant can be determined with an optimal rule curve. The method is based on the following fact: raising the level of a reservoir feeding a powerplant is profitable as long as the gain due to the higher head is greater than the loss due to the additional spillage. There consequently exists a reservoir level $S_{t}$ at time $t$ at which the expected energy generation is maximized. The optimal rule curve is the curve that links the optimal levels $S_{1}, S_{2}, \ldots, S_{T}$. The paper also shows how to use this curve to operate the reservoir. The problem is more complicated for several reservoirs in series since the optimal level of each reservoir is a function of the levels of all the other reservoirs. The paper shows how to solve the problem.
\end{abstract}

Keywords: multi-reservoir operation, rule curves, stochastic dynamic programming, synthetic inflow scenarios.

\section{Introduction}

The problem of determining the optimal weekly operating policy of several hydroelectric powerplants in series has been dealt with in the past, but always in an approximate way. So far, nobody has succeeded in finding a global feedback solution to the problem, i.e., an operating policy that depends on the content of each reservoir and its inflows. Arvanitidis and Rosing [2], for instance, built an aggregate model for all the installations on the river so as to have only one reservoir to manage. The optimal operating policy of the aggregate reservoir, determined with Stochastic Dynamic Programming (SDP), gives the total amount of potential energy to release from the reservoirs each week, but not the 
amount of water to release from each reservoir. Turgeon [13] came up with better results because he used two state variables in his SDP problem: the water content of a reservoir and the total energy content of the downstream reservoirs. He established an operating policy for each reservoir that is a function of these two state variables. However, since this operating policy is not a function of the inflows, it may not be feasible. Archibald et al. [1] improved the method developed by Turgeon [13] by using four state variables in their SDP problem, and solving the problem with a set of inflow scenarios. The state variables are the water content of reservoir $i$, the total energy content of the reservoirs located upstream from reservoir $i$, the total energy content of the reservoirs located downstream from that reservoir, and an hydrological state containing the label of an inflow scenario. The problem is stochastic because there is a conditional probability of switching from one inflow scenario to another at the beginning of each week. The operating policy determined with this method cannot be claimed to be optimal since it is not a function of all reservoir contents.

Many other optimization techniques have been used to solve the stochastic reservoir optimization problem, notably Stochastic Linear Programming (Loucks [8]), Stochastic Programming with recourse (Seifi and Hipel [12]), Stochastic Dual Dynamic Programming (Pereira and Pinto [10], Jacobs et al. [6]), Gradient Dynamic Programming (Foufoula-Georgiou and Kitanidis [4], Johnson et al. [7], Philbrick and Kitanidis [11]), Stochastic Differential Dynamic Programming (ElAwar et al. [3]) and the Extended Linear Quadratic Gaussian method (Wasimi and Kitanidis [15], Georgakakos and Marks [5], McLaughlin and Velasco [9]).

None of the papers cited above can determine the optimal feedback solution of a large weekly stochastic reservoir management problem. SDP cannot solve problems of more than five state variables in a reasonable time. The SLP method by Loucks [8] does not give any better results. The SDDP method by Pereira and Pinto [10] can solve large problems, but not for a 52-week period. The ELQG method by Wasimi and Kitanidis [15] can also solve large problems, but their solutions are local feedbacks. The aggregation/disaggregation methods by Turgeon [13] and Archibald et al. [1] can solve large problems, but only approximately.

In this paper, there is no approximation and no simplification of the problem. All constraints and nonlinearities are correctly taken into account, and no limit is imposed for the number of reservoirs on the river. The problem is solved with a new method called ORT for Optimal Reservoir Trajectory. This method uses the following fact to solve the problem: raising the level of a reservoir feeding a powerplant is profitable as long as the gain due to the higher head is greater than the loss resulting from the additional spillage. As a result, there is a reservoir level at which the expected energy generation is maximized, and the task of ORT is to find it and use it to operate the reservoir. This level naturally changes throughout the year since the water spillage risk changes. Since the time step is a week in this paper, ORT finds the optimal reservoir level at the beginning of each week. The trajectory determined by these levels is called the "optimal reservoir trajectory". The method can be applied to reservoirs in series and in parallel. 


\section{Problem formulation}

The problem consists in determining the optimal weekly operating policy of $M$ hydroelectric installations in series on a river. Each installation consists of a reservoir feeding a powerplant. The installations are numbered from upstream to downstream. We denote by:

$S_{i, t} \quad$ the content of reservoir $i$ at the beginning of week $t, S_{i, t} \leq S_{i}^{\max }$, where $S_{i}^{\max }$ is the reservoir capacity in $\mathrm{hm}^{3}$;

$Q_{i, t} \quad$ the random variable representing the natural inflow in $\mathrm{hm}^{3}$ to reservoir $i$ in week $t$;

$R_{i, t} \quad$ the discharge in $\mathrm{hm}^{3}$ from reservoir $i$ in week $t$;

$U_{i, t} \quad$ the outflow of powerplant $i$ in week $t, U_{i, t} \leq U_{i}^{\max }$, where $U_{i}^{\max }$ is the powerplant capacity in $\mathrm{hm}^{3}$;

$V_{i, t} \quad$ the spillage from reservoir $i$ in week $t$.

$h_{i, t}\left(S_{i, t}\right)$ the average number of MWh produced by each $\mathrm{hm}^{3}$ of water discharged through powerplant $i$ in week $t$ when the reservoir content is equal to $S_{i, t} \mathrm{hm}^{3}$ at the beginning of the week. The function $h_{i, t}\left(S_{i, t}\right)$ is concave.

The optimal operating policy of the $M$ installations is assumed to be the policy that maximizes the generation of electricity. It corresponds to the solution of the following stochastic optimization problem:

$$
\underset{R_{i, t}}{\operatorname{maximize}} E\left\{\sum_{t=0}^{T-1} \sum_{i=1}^{M} h_{i}\left(S_{i, t}\right) \cdot U_{i, t}+F\left(S_{1, T}, S_{2, T}, \ldots, S_{M, T}\right)\right\}
$$

subject to the following constraints:

$$
\begin{array}{cc}
S_{i, t+1}= & S_{i, t}+Q_{i, t}+R_{i-1, t}-R_{i, t} \\
\quad R_{i, t}-U_{i, t}-V_{i, t}=0 & \forall i, t \quad ; S_{i, 0} \text { given, } \quad R_{0, t}=0 \\
0 \leq S_{i, t+1} \leq S_{i}^{\max } & \forall i, t \\
0 \leq U_{i, t} \leq U_{i}^{\max } & \forall i, t \\
V_{i, t} \geq 0 & \forall i, t
\end{array}
$$

where symbol $E$ in (1) represents the mathematical expectation and function $F\left(S_{1, T}, S_{2, T}, \ldots, S_{M, T}\right)$ the value of the water stored in the reservoirs at the end of the time horizon, which is set to one year. Consequently, $T=52$ weeks. When $M$, the number of installations on the river, is large, problem (1)-(6) cannot be solved in a reasonable amount of time because $R_{i, t}^{o p t}$, the optimal discharge from 
reservoir $i$ in week $t$, is a nonlinear function of the variables $S_{1, t}, S_{2, t}, \ldots, S_{M, t}$ and $Q_{1, t}, Q_{2, t}, \ldots, Q_{M, t}$. It is nonlinear because the objective function is nonlinear and there are lower and upper bounds on the variables $S_{i, t}$ and $U_{i, t}$.

The next sections present a very efficient method for solving problem (1)-(6) rapidly, whatever the value of $M$. The method gives a sub-optimal solution, but it has the major advantage of correctly taking into account the cross and serial correlations of the inflows.

\section{Optimal trajectory for a single reservoir}

Let $\bar{S}_{1}^{\text {opt }}=\left\{S_{1,1}^{\text {opt }}, S_{1,2}^{\text {opt }}, \ldots, S_{1, T}^{\text {opt }}\right\}$ represent the optimal trajectory of reservoir 1 , which, for the moment, is assumed to be the only reservoir in the system. This trajectory is said to be optimal because the expected energy generation is maximized when the powerplant discharge is set to:

$$
U_{1, t}=\operatorname{MIN}\left(U_{1}^{\max }, \operatorname{MAX}\left(0, S_{1, t}+Q_{1, t}-S_{1, t+1}^{\text {opt }}\right)\right) \quad \text { for } \quad t=0,1, \ldots, T-1
$$

The optimal operating policy, according to equation (7), is to maintain the reservoir level as close as possible to the trajectory $\bar{S}_{1}^{\text {opt }}$, without spilling water unnecessarily. The trajectory $\bar{S}_{1}^{\text {opt }}$ can be determined as follows.

Suppose that there are $N$ one-year-long inflow scenarios for reservoir 1 , and let $Q_{1, t}^{n}$ denote the inflow in week $t$ corresponding to scenario $n$. There will be no spillage with this scenario in week $T-1$ if the following inequality is satisfied:

$$
S_{1, T-1}+Q_{1, T-1}^{n}-U_{1}^{\max } \leq S_{1}^{\max }
$$

Let $S_{1, T-1}^{n}$ denote the largest value of $S_{1, T-1}$ that satisfies inequality (8).

$$
S_{1, T-1}^{n}=\operatorname{MIN}\left(S_{1}^{\max }, S_{1}^{\max }+U_{1}^{\max }-Q_{1, T-1}^{n}\right)
$$

It is easy to show that there will be no spillage in weeks $T-2$ and $T-1$ with scenario $n$ if:

$$
S_{1, T-2}+Q_{1, T-2}^{n}-U_{1}^{\max } \leq S_{1, T-1}^{n}
$$

There will be no spillage in week $T-2$ since $S_{1, T-1}^{n} \leq S_{1}^{\max }$, and no spillage will occur in week $T-1$ since $S_{1, T-1} \leq S_{1, T-1}^{n}$. There will consequently be no spillage in these two weeks if $S_{1, T-2}$ is smaller than or equal to: 


$$
S_{1, T-2}^{n}=\operatorname{MIN}\left(S_{1}^{\max }, S_{1, T-1}^{n}+U_{1}^{\max }-Q_{1, T-2}^{n}\right)
$$

By proceeding by induction, it can be shown that no spillage will occur in weeks $t, t+1, \ldots, T-1$ with scenario $n$ if $S_{1, t}$ is smaller than or equal to:

$$
S_{1, t}^{n}=\operatorname{MIN}\left(S_{1}^{\max }, S_{1, t+1}^{n}+U_{1}^{\max }-Q_{1, t}^{n}\right) \quad \text { where } \quad S_{1, T}^{n}=S_{1}^{\max }
$$

and the reservoir discharge is set to:

$$
U_{1, t}=\operatorname{MIN}\left(U_{1}^{\max }, \operatorname{MAX}\left(0, S_{1, t}+Q_{1, t}^{n}-S_{1, t+1}^{n}\right)\right)
$$

Solving equation (12) backwards in time for each one of the $N$ inflow scenarios gives $N$ different trajectories for the reservoir, and more specifically $\bar{S}_{1}^{n}=\left\{S_{1,0}^{n}, S_{1,1}^{n}, \ldots, S_{1, T}^{n}\right\}$ for $n=1,2, \ldots, N$. Let:

$$
S_{1, t}^{\text {low }}=\operatorname{MIN}\left(S_{1, t}^{n} ; n=1,2, \ldots, N\right) \quad \text { for } \quad t=0,1, \ldots, T
$$

The trajectory $\bar{S}_{1}^{\text {low }}=\left\{S_{1,0}^{\text {low }}, S_{1,1}^{\text {low }}, \ldots, S_{1, T}^{\text {low }}\right\}$ can be shown to correspond to the lower envelope of the $N$ trajectories determined with (14). There will consequently be no spillage in the next $T$ weeks if:

- $\quad S_{1,0} \leq S_{1,0}^{\text {low }}$

- $U_{1, t}=\operatorname{MiN}\left(U_{1}^{\max }, \operatorname{MAX}\left(0, S_{1, t}+Q_{1, t}-S_{1, t+1}^{\text {low }}\right)\right)$ for $t=0,1, \ldots, T$;

- the inflows in these weeks correspond to one of the $N$ scenarios.

This statement is valid however only if $S_{1, t}^{\text {low }} \geq 0 \quad \forall t$, which is not always the case. If the reservoir is small, there may be years where spillage will occur during the spring no matter what operating policy is followed. If this happens with scenario $n$, for instance, the value of $S_{1, t}^{n}$ will be negative at the beginning of the spring freshet, and so will be $S_{1, t}^{\text {low }}$. However, since the reservoir content cannot be negative, this scenario must be removed from equation (14) before plotting the reservoir envelope. In other words, the lower envelope must be determined with the following equation instead of (14).

$$
S_{1, t}^{\text {low }}=\operatorname{MIN}\left(S_{1, t}^{n} ; n \in \Psi\right) \quad \text { for } \quad t=0,1, \ldots, T
$$


where

$$
\Psi=\left\{n ; S_{1, t}^{n} \geq 0 \quad \forall t\right\}
$$

The scenarios with a negative value of $S_{1, t}^{n}$ that have been removed from set $\Psi$ will naturally produce spillages. The amount of water spilled can be determined by simulating the operation of the reservoir with the following algorithm:

1) Set $G^{*}=V^{*}=0$

2) Set $n=1$

3) Set $t=0$

4) Set $U_{1, t}=\operatorname{MIN}\left(U_{1}^{\max }, \operatorname{MAX}\left(0, S_{1, t}+Q_{1, t}^{n}-S_{1, t+1}^{\text {low }}\right)\right)$

5) Set $G^{*}=G^{*}+h_{1}\left(S_{1, t}\right) \cdot U_{1, t}$

$$
\begin{gathered}
S_{1, t+1}=S_{1, t}+Q_{1, t}^{n}-U_{1, t} \\
V^{*}=V^{*}+\operatorname{MAX}\left(0, \quad S_{1, t+1}-S_{1}^{\max }\right) \\
S_{1, t+1}=\operatorname{MIN}\left(S_{1, t+1}, \quad S_{1}^{\max }\right)
\end{gathered}
$$

6) Set $t=t+1$. If $t<T$, go back to step 4 .

7) Set $n=n+1$. If $n \leq N$ set $S_{1,0}=S_{1, T}$ and go back to step 3 .

8) Stop.

The amount of spillage in the simulation of a period of $N$ is determined by equation (24) and is therefore equal to $V^{*} \mathrm{hm}^{3}$. The generation of hydroelectric energy, calculated with equation (22), is equal to $G^{*}$ MWh, which means that the expected annual energy generation is approximately equal to $G^{*} / N$ MWh. This generation may initially be small since trajectory $\bar{S}_{1}^{\text {low }}$, used in equation (21), was designed to minimize spillage and not to maximize generation. If $\bar{S}_{1}^{\text {low }}$ is raised, spillages would increase, but the value of $h_{1}\left(S_{1, t}\right)$ in (22) would increase also, so that the total generation might increase. The optimal solution consists in raising $\bar{S}_{1}^{\text {low }}$ for as long as the value of $G^{*}$ increases, which can be done in the following way. Suppose that trajectory $i$ is the trajectory in set $\Psi$ with the lowest point. If this trajectory is removed from $\Psi$ before solving (16), the new envelope $\bar{S}_{1}^{\text {low }}$ will be higher than the preceding one in some weeks, and so will the value of $h_{1}\left(S_{1, t}\right)$. The value of $V^{*}$ will of course increase because scenario $i$ will now produce spillages, which was not the case before. When the generation $G^{*}$ increases also, another trajectory is removed from $\Psi$, more specifically the one with the lowest point. The procedure of removing the 
scenario with the lowest point from $\Psi$, building a new lower envelope with (16) and determining generation $G^{*}$ with algorithm (18)-(28) is repeated as long as $G^{*}$ increases. The envelope $\bar{S}_{1}^{\text {low }}$ that maximizes the generation is optimal, and can therefore be referred to by $\bar{S}_{1}^{\text {opt }}$.

\section{Optimal trajectories for several reservoirs}

Turgeon [14] has shown that three trajectories are required for controlling spillages at two reservoirs in series: one for each reservoir and a third for the two reservoirs combined. The paper in fact shows that there will be no spillage at the two reservoirs in week $t$ if:

$$
\begin{gathered}
S_{1, t}+Q_{1, t}-U_{1}^{\max } \leq S_{1}^{\max } \\
S_{2, t}+Q_{2, t}-U_{2}^{\max } \leq S_{2}^{\max } \\
\hat{S}_{1,2, t}+\hat{Q}_{1,2, t}-U_{2}^{\max } \leq \hat{S}_{1,2}^{\max }
\end{gathered}
$$

where

$$
\hat{S}_{i, j, t}=\sum_{m=i}^{j} S_{m, t}, \quad \hat{S}_{i, j}^{\max }=\sum_{m=i}^{j} S_{m}^{\max } \quad \text { and } \quad \hat{Q}_{i, j, t}=\sum_{m=i}^{j} Q_{m, t}
$$

For three reservoirs in series, there will be no spillage in week $t$ if six inequalities are respected: one, similar to (29), for each of the three reservoirs and three, similar to (31), for the combined reservoirs 1 and 2, 2 and 3, and 1,2 and 3. The following $M \times(M+1) / 2$ inequalities will have to be respected for $M$ reservoirs in series:

$$
\hat{S}_{i, j, t}+\hat{Q}_{i, j, t}-U_{j}^{\max } \leq \hat{S}_{i, j}^{\max } ; \quad i=1, \ldots, M ; \quad j=i, \ldots, M
$$

The optimal trajectory of reservoir $i, \bar{S}_{i}^{\text {opt }}$, can be determined with the procedure described in section 3 for reservoir 1 . The optimal trajectory of reservoirs 1 and 2 combined, $\bar{S}_{1,2}^{\text {opt }}$, cannot, however, be determined with the same procedure since it is not known how $\hat{S}_{1,2, t}^{\text {low }}$ is divided between the two reservoirs and, hence, what the values of $h_{1}\left(S_{1, t}\right)$ and $h_{2}\left(S_{2, t}\right)$ are. But since the objective of the problem is to maximize generation, the values of $S_{1, t}$ and $S_{2, t}$ should be adjusted to:

$$
\text { maximize } h_{1}\left(S_{1, t}\right) \cdot U_{1, t}^{\text {mean }}+h_{2}\left(S_{2, t}\right) \cdot U_{2, t}^{\text {mean }}
$$

subject to: 


$$
\begin{gathered}
S_{1, t}+S_{2, t}=\hat{S}_{1,2, t}^{\text {low }} \\
0 \leq S_{i, t} \leq S_{i, t}^{\text {low }} \quad i=1,2
\end{gathered}
$$

where $U_{i, t}^{\text {mean }}$ refers to the mean outflow of powerplant $i$ in week $t$. When the value of $U_{i, t}^{\text {mean }}$ is not initially known, one can start by solving the problem with $U_{1, t}^{\text {mean }}=Q_{1, t}^{\text {mean }}$ and $U_{2, t}^{\text {mean }}=Q_{1, t}^{\text {mean }}+Q_{2, t}^{\text {mean }}$, where $Q_{i, t}^{\text {mean }}$ represents the mean natural inflow to reservoir $i$ in week $k$, and change these values afterwards for the mean outflows determined by simulating the reservoir operation over a period of $N$ years. The procedure must of course be repeated until the values of $U_{1, t}^{\text {mean }}$ and $U_{2, t}^{\text {mean }}$ no longer change.

It follows from the above discussion that the discharge from the two reservoirs in week $t$ should be adjusted to:

$$
\text { maximize } \quad h_{1}\left(S_{1, t+1}\right) \cdot U_{1, t+1}^{\text {mean }}+h_{2}\left(S_{2, t+1}\right) \cdot U_{2, t+1}^{\text {mean }}-P E N
$$

subject to constraints (2)-(6) and those following:

$$
\begin{array}{cc}
S_{1, t+1}+S_{2, t+1}-W_{1,2} \leq & \hat{S}_{1,2, t+1}^{l o w} \\
S_{i, t+1}-W_{i, i} \leq S_{i, t+1}^{l o w} & i=1,2 \\
W_{i, j} \geq 0 & \forall i, j
\end{array}
$$

where

$$
P E N=b\left(W_{1,1}^{2}+W_{2,2}^{2}+W_{1,2}^{2}\right)+c\left(V_{1, t}^{2}+V_{2, t}^{2}\right)
$$

The dummy variable $W_{i, j}$ is greater than zero only when the lower envelope for reservoirs $i$ to $j$ is exceeded. The variable $P E N$ in (41) gives the penalty cost for exceeding the lower envelopes and spilling water. The role of $P E N$ is to force the optimizer to search for a solution that respects constraints (29)-(31). If no such solution exists, the square costs in $P E N$ help keeping the violations small. The penalty cost for spilling water is necessary to prevent unnecessary spillages. With that cost, spillage occurs only when there is no other solution. The values of parameters $b$ and $c$ in (41) are positive and $c>b$.

The operation of the two reservoirs can now be simulated with algorithm (18)-(28) if steps 4 and 5 are replaced by:

4) Solve problem (37)-(41) to determine $U_{i, t}, V_{i, t}$ and $S_{i, t+1}, i=1,2$.

5) Set $G^{*}=G^{*}+h_{1}\left(S_{1, t}\right) \cdot U_{1, t}+h_{2}\left(S_{2, t}\right) \cdot U_{2, t}$ 


$$
V^{*}=V^{*}+V_{1, t}+V_{2, t}
$$

and $S_{1,0}=S_{1, T}$ in step 7 is replaced by $S_{i, 0}=S_{i, T}, i=1,2$.

Problem (37)-(41) and equations (42) and (43) can be extended to any number of reservoirs. The procedure consists in determining, first, the optimal trajectory of each reservoir alone, followed by every combination of two neighboring reservoirs, three neighboring reservoirs, and so forth.

\section{References}

[1] Archibald, T.W., McKinnon, K.I.M. \& Thomas, L.C., An aggregate stochastic dynamic programming modef of multiple reservoir systems, Water Resources Research, 33(2), pp. 333-340, 1997.

[2] Arvanitidus, N.V. \& Rosing, J., Composite representation of a multireservoir hydroelectric power system, IEEE Trans. on Power Appar. and systems, 89(2), pp. 319-326, 1970.

[3] El-Awar, F.A., Labadie, J.W. \& Ouarda, T.B.M.J., Stochastic differential dynamic programming for multi-reservoir system control, Stochastic Hydrology and Hydraulics, 12, pp. 247-266, 1998.

[4] Foufoula-Georgiou, E. \& Kitanidis, P.K., Gradient dynamic programming for stochastic optimal control of multidimentional water resources systems, Water Resources Research, 24(8), pp. 1345-1359, 1988.

[5] Georgakakos, A. \& Marks, D., A new method for the real-time operation of reservoir systems, Water Resources Research, 23(7), pp. 1376-1390, 1987.

[6] Jacobs, J., Freeman, G., Grygier, J., Morton, D., Schultz, G. Staschus, K. \& Stedinger, J., SOCRATES: a system for scheduling hydroelectric generation under uncertainty, Annals of Operations Research, 59, pp. 99133, 1995.

[7] Johnson, S.A., Stedinger, J.R., Shoemaker, C.A., Li, Y. \& Tejada-Guibert, J.A., Numerical solution of continuous-state dynamic programs using linear and spline interpolation, Operation Research, 41(3), pp. 484-500, 1993.

[8] Loucks, D.P., Computer models for reservoir regulations, J. Sanit. Eng, Div, Am. Soc. Civ. Eng., 94(SA4), pp. 657-669, 1968.

[9] McLaughlin, D. \& Velasco, H.L., Real-time control of a system of large hydropower reservoirs, Water Resources Research, 26(4), pp. 623-635, 1990.

[10] Pereira, M.V.F. \& Pinto, L.M.V.G., Stochastic optimization of a multireservoir hydroelectric system - a decomposition approach, Water Resources Research, 21(6), pp. 779-792, 1985.

[11] Philbrick, C.R. \& Kitanidis, P.K., Improved dynamic programming methods for optimal control of lumped-parameter stochastic systems, Operations Research, 49(3), pp. 398-412, 2001. 
[12] Seifi, A. \& Hipel, K., Interior-point method for reservoir operation with stochastic inflows, J. Water Resour. Plan. Manage., 127(1), pp. 48-57, 2001.

[13] Turgeon, A., A decomposition method for the long-term scheduling of reservoirs in series, Water Resources Research, 17(6), pp. 1565-1570, 1981.

[14] Turgeon, A., Optimal daily operation of reservoirs subject to probabilistic flood constraints, River Basin Management II, C.A. Brebbia (ed.), W.I.T. Press, Southampton, UK, pp. 153-162, 2003.

[15] Wasimi, S. \& Kitanidis, P., Real-time forecasting and daily operation of a multireservoir system during floods by linear quadratic Gaussian control, Water Resources Research, 19(6), pp. 1511-1522, 1983. 